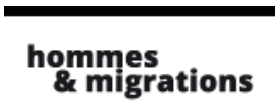

\section{Hommes \& migrations}

Revue française de référence sur les dynamiques

migratoires

$1324 \mid 2019$

Religion et discrimination

\title{
Persona grata
}

\section{Ingrid Jurzak}

\section{QpenEdition \\ Journals}

\section{Édition électronique}

URL : https://journals.openedition.org/hommesmigrations/8989

DOI : 10.4000/hommesmigrations.8989

ISSN : 2262-3353

\section{Éditeur}

Musée national de l'histoire de l'immigration

\section{Édition imprimée}

Date de publication : 1 janvier 2019

Pagination : 78-79

ISBN : 978-2-919040-44-5

ISSN : $1142-852 X$

\section{Référence électronique}

Ingrid Jurzak, «Persona grata », Hommes \& migrations [En ligne], 1324 | 2019, mis en ligne le 01 janvier 2019, consulté le 06 janvier 2022. URL : http://journals.openedition.org/hommesmigrations/8989 ; DOI : https://doi.org/10.4000/hommesmigrations.8989 


\title{
PORTFOLIO
}

\section{Persona grata}

\section{Ingrid Jurzak,}

chargée de l'étude et de la gestion de la collection du MAC VAL-

Musée d'art contemporain du Val-de-Marne et commissaire de l'exposition.

\author{
«Il n'est rien que l'homme redoute davantage \\ que le contact de l'inconnu. »
}

Elias Canetti, Masse et puissance, 1960.

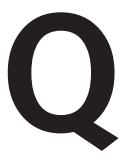

uand un défi humanitaire sans précédent se joue aux portes de l'Europe, le MAC VAL - Musée d'art contemporain du Val-de-Marne et le Musée national de l'histoire de l'immigration s'associent et proposent Persona grata : un regard croisé dans leurs collections afin d'envisager le sens et la place de l'hospitalité dans notre société.

Le MAC VAL a été inauguré en novembre 2005, ouvrant à Vitry-sur-Seine les portes d'un édifice conçu par les architectes Denise Duhart et Jacques Ripault dédié à la rencontre entre une population et l'art de son temps. Si le principe de construction du musée était voté en 1990, la volonté départementale de constituer une collection d'art contemporain, dans une dynamique de soutien aux artistes, d'émancipation culturelle et de cohésion sociale, remonte au début des années 1980. À la faveur des lois de décentralisation, les élus du département du Val-de-Marne choisissaient d'inscrire la culture au cœur de leur politique sociale et d'initier un Fonds départemental d'art contemporain. Le processus de constitution du fonds, principalement par l'acquisition d'œuvres, était naturellement ouvert sur le monde, mais également attentif aux productions des artistes présents sur le territoire. Ce dernier comptait en effet d'importantes communautés d'artistes réunies autour d'Antonio Segui, Julio Le Parc, Pierre Buraglio ou Jacques Monory, et certaines villes, telles Arcueil et Cachan, accueillaient de nombreux créateurs exilés en France après avoir fui les régimes autoritaires d'Amérique latine ou d'Europe de l'Est. L'art en France de 1950 à nos jours définit désormais le projet scientifique et culturel du MAC VAL. La collection du musée et sa programmation accordent ainsi une large place aux expressions visuelles françaises comme à celles d'artistes étrangers travaillant en France. La collection compte aujourd'hui près de 2500 œuvres et réunit plus de 400 artistes. Par la mise en œuvre d'une expertise et d'un travail de veille permanent, elle répond à une exigence de représentation et de pédagogie. Afin de permettre une large présentation de ses œuvres, le MAC VAL renouvelle régulièrement les expositions de sa collection imaginées et organisées en parcours thématiques. Chacun de ces accrochages place le visiteur et la communauté humaine au cœur de développements artistiques et offre des points de rencontre avec la vie des gens ou l'actualité. Les thèmes traités relèvent ainsi tant d'une histoire de l'art en résonance avec son temps que de sujets de société. Aussi, après avoir interrogé les notions de l'exil, du souvenir et des utopies, était-il naturel d'envisager celle de l'hospitalité et d'accepter avec enthousiasme et conviction de traiter ce motif que nous proposait le Palais de la Porte Dorée. 
L'accroissement des flux migratoires occupe, en effet, une place grandissante dans un débat public dont l'influence tend à bousculer les fondements de nos valeurs constitutionnelles. Une dynamique organisée de contrôle des frontières semble opérer un renversement irréversible du devoir d'hospitalité, tandis que des mobilisations associatives et citoyennes s'amplifient, osant si besoin la désobéissance civile, pour soutenir et accueillir les plus fragilisés.

À leur manière, les artistes s'emparent de ces sujets. Certains témoignent, tandis que d'autres produisent des œuvres qui, avec la distance de l'art, autorisent une appréhension autre de notre réalité contemporaine. Les œuvres réunies au MAC VAL pour Persona grata dévoilent des frontières métaphoriques et esquissent une cartographie symbolique des zones et groupes humains les plus sensibles. Elles proviennent pour la plupart des collections de nos deux institutions, et pour quelques autres, de la générosité des artistes, de leurs galeries, d'autres musées comme de fonds régionaux ou départementaux d'art contemporain.

Ces œuvres dévoilent des murs, laissant apparaître un versant hostile au cœur de la thématique, mais pourfendent également le caractère arbitraire et autoritaire des frontières, à l'instar de U.N. Camouflage, installation de la coopérative Société Réaliste, véritable point de jonction artistique et visuel du Palais de la Porte Dorée au MAC VAL, qui accueille les visiteurs sur le parvis des deux institutions. Elles évoquent également les sources de l'hospitalité au sein de notre histoire et de notre territoire, à travers mythes, philosophies et valeurs républicaines, et témoignent de son effectivité passée au sein d'une société multiculturelle, riche des héritages variés qui la composent. Une variété d'images révèle à quel point le départ est impératif tandis que le déplacement n'est que péril et la destinée incertaine.

Le déracinement et l'errance s'y incarnent dans des formes et des figures poétiques et universelles, quand elles ne sont pas délibérément provocatrices et osent la voie de l'humour pour éveiller les consciences. De leurs expériences ou appréhensions personnelles de l'exil, les artistes livrent des motifs oscillant entre ouverture et fermeture, liberté et empêchement, résignation et résistance. Ils rappellent que, si le secours n'est pas hospitalité, des abris précaires, organisés ou improvisés, s'imposent néanmoins comme possibles prémices d'une vie nouvelle. Leurs œuvres nous amènent à reconnaître que «nulle part est un endroit ${ }^{1}$ » et que le soin et la bienveillance sont des tensions naturelles que nous pouvons favoriser en résistance à l'invisibilité de l'Autre. Avec les artistes, essayons d'entrevoir une hospitalité politique que légitime notre responsabilité éthique.

Pour l'écrire simplement, l'exposition Persona grata est une tentative de préserver, par l'art, le désir d'autrui. "

Avec les œuvres d'Eduardo Arroyo, Kader Attia, Renaud Auguste-Dormeuil, Marcos Avila Forero, Laëtitia Badaut Haussmann, Bertille Bak, Richard Baquié, Taysir Batniji, Ben, Bruno Boudjelal, David Brognon \& Stéphanie Rollin, Mark Brusse, Pierre Buraglio, Mircea Cantor, Étienne Chambaud, Kyungwoo Chun, Clément Cogitore, Philippe Cognée, Delphine Coindet, Matali Crasset, Julien Discrit, Thierry Fontaine, Jochen Gerz, Ghazel, Marie-Ange Guilleminot, Mona Hatoum, Eric Hattan, Laura Henno, Pierre Huyghe, Emily Jacir, Yeondoo Jung, Bouchra Khalili, Kimsooja, Claude Lévêque, M/M, Lahouari Mohammed Bakir, Jean-Christophe Norman, Lucy Orta, Bernard Pagès, Cécile Paris, Philippe Parreno, Yan Pei-Ming, Mathieu Pernot, Jacqueline Salmon, Bruno Serralongue, Esther Shalev-Gerz, Société Réaliste, Djamel Tatah, Barthélémy Toguo, Patrick Tosani, Sabine Weiss. 